\title{
An Energy Loss Calculating Method for Wind Power System Based on the Shape Factor
}

\author{
Bo RUAN ${ }^{1}$, Junjie QIAN², Dahai YOU ${ }^{2}$, Tingting $\mathrm{HOU}^{1}, \mathrm{Xi} \mathrm{CHEN}^{1}$, Zhen MEI ${ }^{2}$ \\ ${ }^{1}$ Research Institute of Economics and Technology, Hubei Electric Power Company Limited, Wuhan, Hubei Province, China 430077 \\ 2 State Key Laboratory of Advanced Electromagnetic Engineering and Technology, Huazhong University of Science and Technology, \\ Wuhan, Hubei Province, China 430074
}

\begin{abstract}
The large-scale access of the new energy makes various changes to the power system characteristics. For example, the acute volatility of the new energy such as wind power and photovoltaic energy makes the power-losscalculation for the system more complex. The traditional typical daily method is inaccurate when used in new energy system because of its overlook of the generator output volatility. This paper proposes a new power-loss-calculation method for wind power system which based on the shape factor and gain a more accurate result. On the basis of this new calculating method, finds that the shape factor of the wind power plant for an hour period usually falls in a certain range. Therefore, proposes to directly use the expectation of shape factor in whole year to compute the annual energy loss with at least two values and once power flow calculation. And the acceptable relative error proves its large engineering practicability.
\end{abstract}

\section{Introduction}

With the development of global economy, human demand for energy is growing while the primary energy such as fossil fuel is drying up. As a result, the new energy like wind power gains a national encouragement for its renewability and cleanliness. However, the access of the new energy will have an influence on the energyloss-calculation for the power system because wind power and photovoltaic energy have features of randomness, volatility and regionalism [1-3]. The traditional energy-loss-calculation method is the typical daily method, whose basic thinking is to calculate the electric energy loss of the representative day first, and then estimate the whole periodic energy loss [4]. This method entirely ignores the volatility of generation and load, considers the generator output and load remain unchanged within one hour and the power loss of everyday in the whole period is the same. Obviously, the wind power plant whose output changes sharply every minute is not suitable for this traditional method. However, if we adequately take the volatility into account, amount of measured data and repeated power flow calculation are required. It's too fussy and timeconsuming to be applied in the practical projects. As the installed capacity of the new energy is growing, seeking an efficient and simple energy-loss-calculation method for wind power system is worth studying.

This paper proposes an energy loss calculating method for wind power system based on the shape factor, it uses hourly shape factors of the whole period to correct the wind power output curve and gain a more accurate loss result. In the further study, finds the hourly shape factors of wind power plant usually focus on a small area and considers to calculate the annual energy loss with the expectation of the whole year shape factors. In this way, it at least only needs to conduct once power flow calculation and has a small relative error.

\section{The typical daily method for energy loss calculation}

The traditional power loss calculating method is typical daily method. Its basic thinking is to calculate the energy loss of the typical day first, and then reckon the whole periodic energy loss through the typical-day's.

Using this method to calculate the monthly power loss:

(1). Consider the generator output and the load remain unchanged within every hour in the representative day. Use $I_{t, l}$ and $V_{t, m}$ to represent the current of line $l$ and the voltage of node $m$ in hour $t, R_{t . l}$ and $G_{t, m}$ to represent line resistance and node conductance and the energy loss of representative day $\square A_{d}$ is:

$$
\Delta A_{d}=\left[\sum_{l=1}^{L}\left(3 \sum_{t=1}^{24} I_{t, l}^{2} R_{t, l}\right)+\sum_{m=1}^{M} \sum_{t=1}^{24} V_{t, m}^{2} G_{t, m}\right]
$$

(2). Consider the watt hour meter is more accurate than the power meter. When these two meters are inconsistent, correct the power graph by apportioning the electric quantity to per hour according to the load and generator output curve on the typical day. 
(3). Use the corrected load and generator output to compute the line current and node voltage through power-flow calculation. And then take the result into the formula (1) to calculate the energy loss of the representative day.

(4). Consider the daily power loss is the same as the representative day within the whole month and as a result, multiply the power loss of typical day by the number of days in that month to get the power loss of the whole month.

The specific formulas and details of the typical daily method are not elaborated in this paper and they can be referred to in reference [5].

\section{Energy loss calculation for wind power system based on the shape factor}

\subsection{Equivalent power method and the shape factor}

Equivalent power method is a well-known electrical energy calculating method uses the average power because of its usage of the shape factor. For one line, the power loss within time $T$ is:

$$
\Delta A=3 \int_{0}^{T} I^{2} R d t=3 I_{e q}^{2} R T=\frac{P_{e q}^{2}+\mathrm{Q}_{e q}^{2}}{V^{2}} R T
$$

where, $I_{e q}, P_{e q}$ and $Q_{e q}$ represent the equivalent value of the current, the active power and the reactive power respectively. The equivalent value is actually the rootmean-square value. The equivalent value of the current, the active power and the reactive power can be expressed by their average value as:

$$
\begin{aligned}
& I_{e q}=G I_{a v} \\
& P_{e q}=K P_{a v} \\
& Q_{e q}=L Q_{a v}
\end{aligned}
$$

where, $G, K$ and $L$ are shape factors of the load curve $I(t)$, $P(t)$ and $Q(t)$ respectively.

The energy loss can be calculated by average power through the shape factor, and the average values are more available and accurate. The studies on shape factor show that it is decided by the form of the load curve. Actually, it is a reflection for the volatility of the load curve, the greater the volatility, the bigger the shape factor. It can be gain through mathematical derivation that the value range of the shape factor $K$ is:

$$
1 \leq K \leq \frac{1+\alpha}{2 \sqrt{\alpha}}
$$

where, $\alpha$ is the ratio of the minimum load to the maximum load called minimum load ratio. The concrete details can be referred to in reference [6].

\subsection{An energy loss calculating method for wind power system based on the shape factor}

The typical daily method has two assumptions that ignore the volatility of the generator output. First, considers the energy loss of everyday in the whole period is the same.
The other is considering the generator output and the load remain unchanged within every hour.

This two assumptions can improve the calculation efficiency, but can also bring prodigious calculating error when applied in wind power system because of its acute volatility. As a result, it should be improved. First, for the representative day, this paper proposes the concept of wind power output expectation graph and it can be calculated as equation (5) below. The method collects the data of the wind power output per hour per day in the whole calculating period. Then the superposition of the data in per hour is divided by the number of days to acquire the expectation of wind power output per hour for that period. The 24 expectations constitute an expectation graph to replace the typical day curve.

$$
P_{m, t}==\frac{\sum_{k=1}^{T_{m}} P_{k, t}}{T_{m}}
$$

where, $P_{m, t}$ is the active power expectation of the wind power output in hour $t, P_{k, t}$ is the active power expectation of the wind power output in hour $t$ on day $k$. $T_{m}$ is the number of days in the whole period.

Secondly, for the output volatility within one hour, this paper brings in the shape factor. Concretely, uses the shape factor of every hour to correct the expression curve gained from the last step for a more accurate energy loss. The steps to calculate the shape factor are as follows:

Consider one hour is a period and the average of the active power is shown in equation (6):

$$
P_{\text {ave }, t}=\frac{\sum_{k=1}^{T_{m}} \sum_{n=1}^{N} P_{k, n, t}}{T_{m} \times N}
$$

The root-mean-square value of the active power is shown in equation (7):

$$
P_{e q, t}=\sqrt{\frac{\sum_{k=1}^{T_{m}} \sum_{n=1}^{N} P_{k, n, t}{ }^{2}}{T_{m} \times N}}
$$

So the shape factor of this period is shown in equation (8):

$$
K_{t}=\frac{P_{e q, t}}{P_{a v e, t}}=\sqrt{\frac{\sum_{k=1}^{T_{m}} \sum_{n=1}^{N} P_{k, n, t}{ }^{2}}{T_{m} \times N}} / \frac{\sum_{k=1}^{T_{m}} \sum_{n=1}^{N} P_{k, n, t}}{T_{m} \times N}
$$

In the equation (6) to (8), $P_{\text {ave, } t}$ is the mean value of active power of wind farms during the period $t, P_{e q, t}$ is the RMS value of active power of wind farms at period $t$, $P_{k, n, t}$ is active power of wind farms at $N$ sampling points during the period $t, N$ is the number of sampling points per hour, and $K_{t}$ is the shape factor of wind farms for the period $t$.

Then multiply the curve of the typical daily expected output by the corresponding shape factor to get the curve of the equivalent output of wind power, as shown in equation (9). Use the equivalent output curve to calculate the energy loss of one day and then estimate the total energy loss of the power system.

$$
P_{t}=K_{t} \cdot P_{m, t}
$$


where, $P_{t}$ is the corrected active power of hour $t, K_{t}$ is the shape factor of hour $t$.

\section{Energy loss quick calculating method for wind power system based on the annual shape factor expectation}

\subsection{Annual shape factor expectation}

Use the shape factor of each hour to corrects the expectation graph can gain an accurate result, but it needs lots of data and many times of power flow calculation. It's time-consuming and complex. When research the output characteristics of a wind power plant, it is found that although the output changes acutely in every hour, the most shape factors of one hour fall in a stable interval. As a result, considers to calculate the total energy loss using the expectation of the whole periodic shape factors. Take one year for example, it at least only needs two values that the annual electricity quantity and the annual shape factor expectation, and to conduct power flow calculation one time to gain the power loss of whole year.

Count the monthly generator output data of Xiangyang wind power plant somewhere and calculate its expectation and equivalent value to constitute the monthly output expectation curve and monthly output equivalence curve. As shown in figure 1. It is obvious that the expectation curve and equivalence curve have a totally the same variation tendency and it means that the shape factor of each hour changes little.

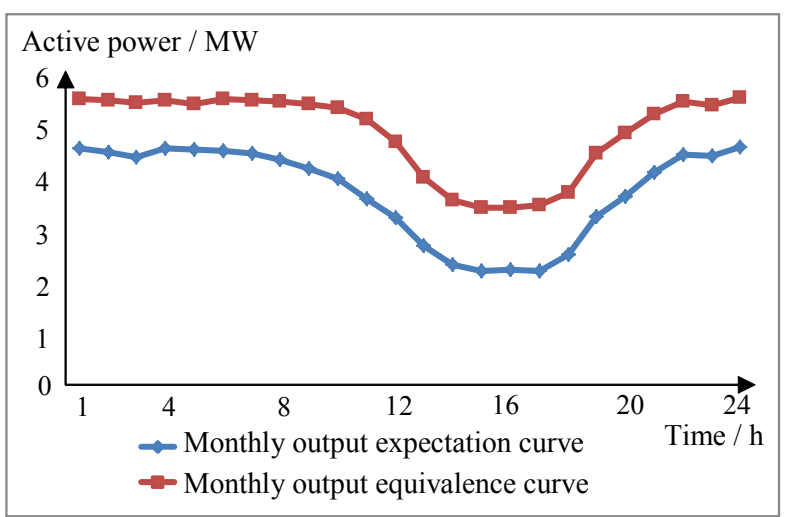

Figure 1. Monthly output expectation curve and monthly output equivalence curve (active power).

In order to further study the characteristics of wind power plant output for seeking an efficient energy loss calculating method, count the whole year output data of the Xiangyang wind power plant with the sampling frequency of 4 points per hour. Calculate the shape factor of each hour and make its frequency distribution histogram as figure 2 .

It can be found from figure 2 that although the shape factors distribute in a wide range in the whole year and the maximum value reaches 2.6 , most values concentrate within a small range. Take the range $[1.2,1.75]$ as a confidence interval, and its confidence coefficient reaches $96 \%$. For the shape factor represents the volatility of the wind power system to some extent, it illustrates that most of the time, the volatility of the wind power system within an hour is close. Consider to use the expectation of whole year shape factor to replace the per hour shape factor for the correction of the expectation curve.

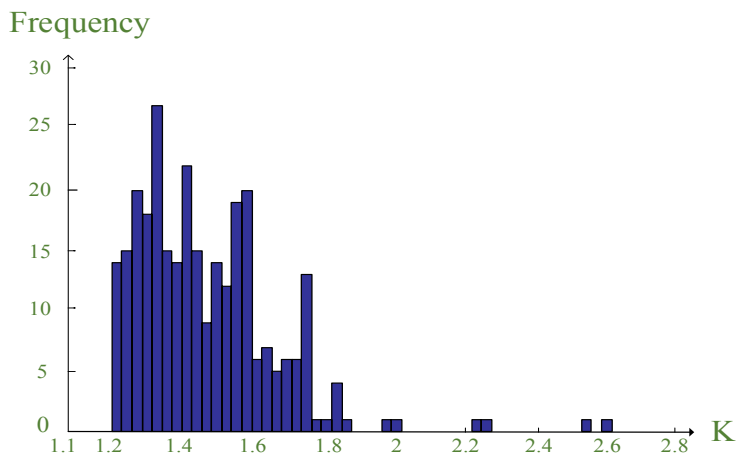

Figure 2. Frequency distribution histogram of shape factors K.

Use the Matlab to match the probability density distribution situation of whole year shape factors, as shown in figure 3.

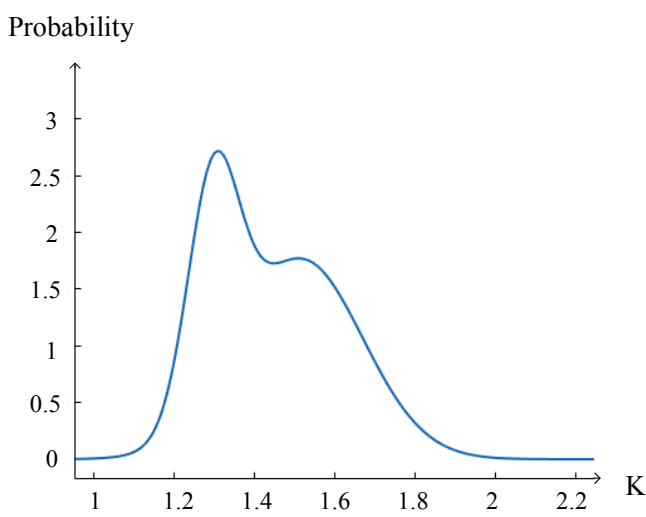

Figure 3. Matched probability density distribution of shape factors $\mathrm{K}$.

As can be seen from the figure 3 that the probability distribution of shape factor presents a tendency of middle high and sides low. It reaches top when $K$ is 1.33 and its form is similar to the normal distribution to some extent, although it is not symmetrical. Calculate the expectation of the whole year shape factors is $K_{a v}=1.43$.

\subsection{Use $K_{\mathrm{av}}$ to calculate annual energy loss}

The structure of a $110 \mathrm{kV}$ main network is shown in figure 4.There are two power generators. One is named Longkou, which is a conventional power generation; the other is Xiangyang wind power plant which has been introduced in section 4.1 and its annual shape factor expectation has been calculated as 1.43 . There are 5 loads and 4 lines in figure 4. 


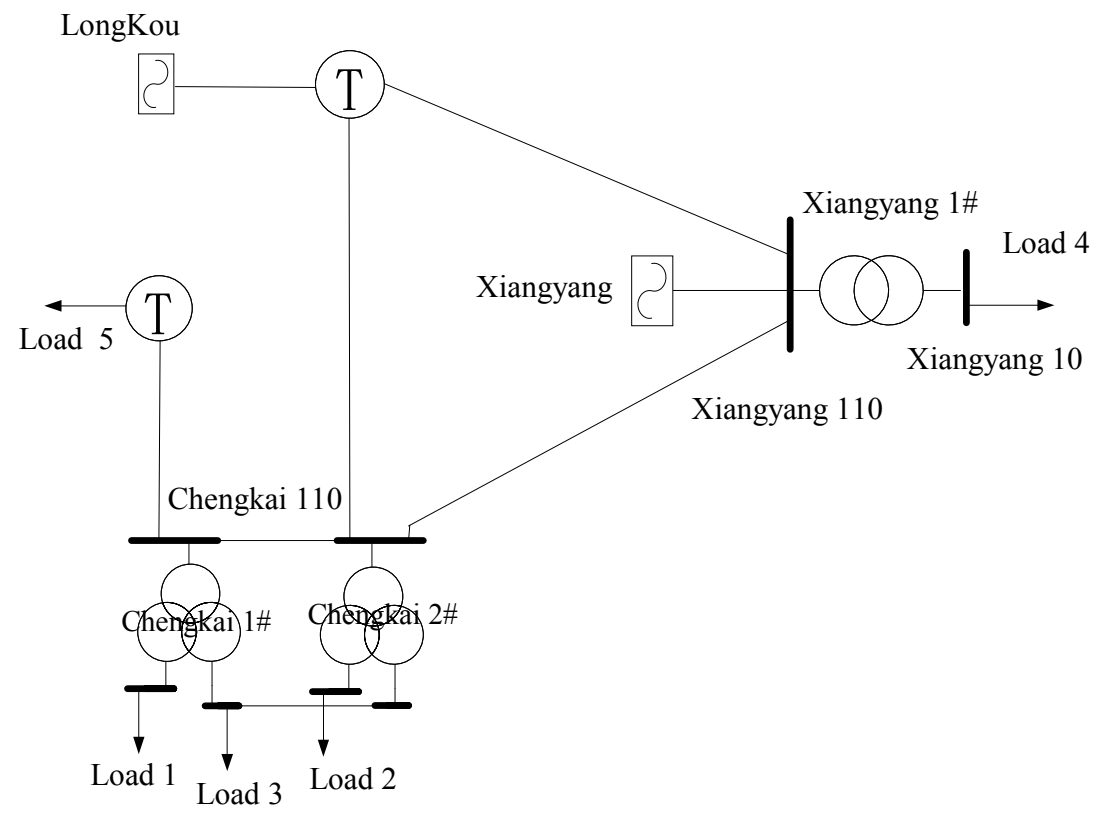

Figure 4. The structure of a $110 \mathrm{kV}$ main network.

Propose four kinds of solutions to calculate the annual energy loss of Xiangyang wind power plant. Use traditional typical daily method, per hour shape factor method and annual shape factor expectation method respectively to calculate the result and compare these methods.

As a reference, use the most complex way to calculate a reference value which is close to the real value. Collect wind power output data of Xiangyang wind farm 4 sampling points per hour. The power flow is calculated 4 times an hour, namely 96 times a day. The energy loss is accumulated day by day to acquire the annual energy loss. The accumulated energy loss value is set to be the reference value.

The reference value calculated by the power flow is 1953.8 MWh.

Solution one: use the traditional typical daily method to calculate the annual energy loss. Choose one day of the year as the representative day and calculate its power loss. Then multiply by the number of days in that year to gain the annual power loss. The load curves of the representative day are shown in figure 5 and figure 6 .

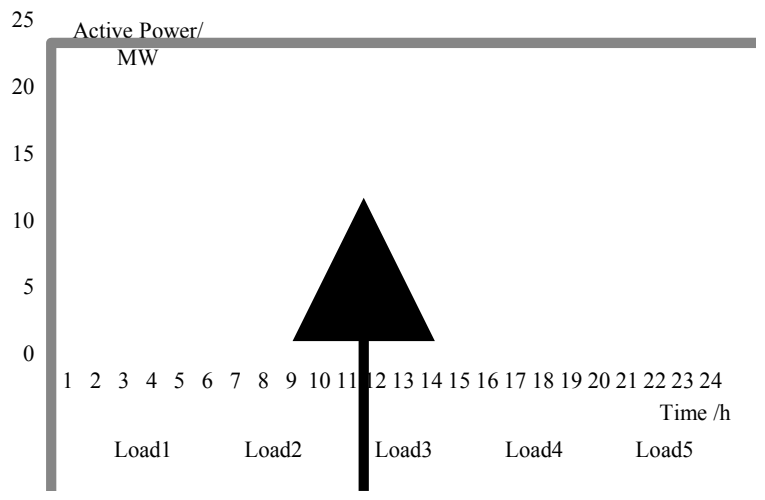

Figure 5. The active load curve of the representative day.

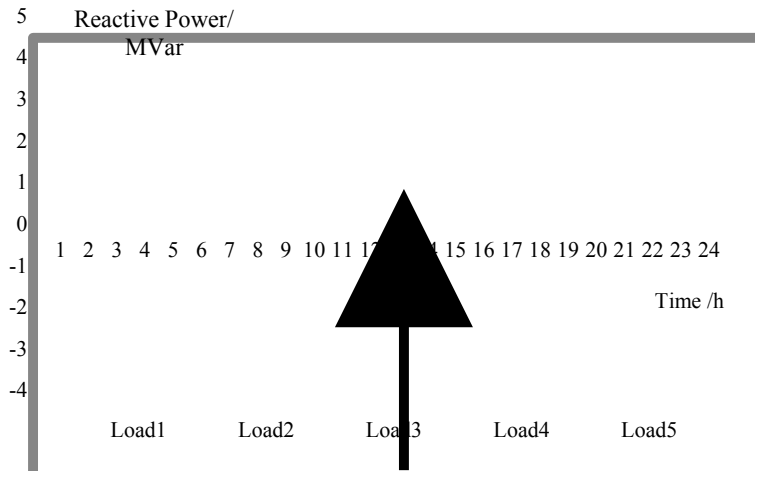

Figure 6. The reactive load curve of the representative day.

Solution two: use the shape factor of per hour to calculate the energy loss. Collect data to calculate the expectation curve of generator output and the shape factor of per hour, and then correct the output expectation curve with the average electricity. Get the curve of the typical daily equivalent output of wind farms for the year by multiplying curve of the typical daily expected output by the corresponding shape factor. And then use the equivalence curve to conduct power flow calculation for the energy loss of the typical day, and finally estimate the annual energy loss of the power system.

Solution three: use the shape factor expectation of whole year to calculate the annual energy loss. Collect data to calculate the expectation curve of generator output and the shape factor expectation of whole year. After correcting the output expectation curve with the average electricity, multiply the curve values by the shape factor expectation of whole year $K_{a v}$ to get an equivalent curve. Then calculate the energy loss of the representative day with the equivalent curve via the power flow calculation. Finally, multiplied with the number of days in the year to calculate annual energy loss. 
Solution four: use annual electricity quantity and annual shape factor expectation to calculate the annual energy loss. Use the annual electricity quantity to calculate the average active power $P_{\text {ave }}$ as equation (10):

$$
P_{\text {ave }}=\frac{A_{y, P}}{T_{y} \times 24}
$$

where, $P_{\text {ave }}$ is the average active power of the wind power system this year, $A_{y, p}$ is the total electricity quantity this year, $T_{y}$ is the number of days in that year.

Correct the $P_{\text {ave }}$ with annual shape factor expectation $K_{a v}$ to gain the equivalent active power $P_{e q}$ as equation (11):

$$
P_{e q}=K_{a v} \times P_{a v e}
$$

where, $P_{e q}$ is the equivalent active power of the wind power system.

Assume that the active power output is the same in the whole year and it is equal to $P_{e q}$. Use the $P_{e q}$ to conduct power flow calculation and gain the energy loss for an hour. As a result, the annual power loss can be calculated as equation (12):

$$
\Delta A_{y 4}=\Delta P \times 24 \times T_{y}
$$

where, $\Delta P$ is the energy loss for an hour, $\Delta A_{y 4}$ is the annual energy loss.

It should be illuminated that the process mode of reactive power is similar to the active power and not be detailed here.

It's obvious from above four solutions that the data and calculating steps needed have a simplified tendency from solution two to solution four. The solution four needs the least data and the simplest calculation steps and if its error is in the allowable range, this method has a strong engineering practicality.

Calculate the annual energy loss with these four solutions above respectively and compared with the reference value. The result is shown in table 1 .

Table 1. Analysis of annual energy loss calculation result.

\begin{tabular}{cccc}
\hline $\begin{array}{c}\text { Calculation } \\
\text { method of } \\
\text { annual energy } \\
\text { loss }\end{array}$ & $\begin{array}{c}\text { Annual } \\
\text { energy loss } \\
\text { (MW) }\end{array}$ & $\begin{array}{c}\text { Reference } \\
\text { value (MW) }\end{array}$ & $\begin{array}{c}\text { Relative } \\
\text { error }\end{array}$ \\
\hline Solution one & 1547.5 & 1953.8 & $20.79 \%$ \\
Solution two & 1846.8 & 1953.8 & $5.47 \%$ \\
Solution three & 1833.4 & 1953.8 & $6.16 \%$ \\
Solution four & 1802.7 & 1953.8 & $7.73 \%$ \\
\hline
\end{tabular}

As shown in table 1, the solution one uses traditional typical daily method and ignores the volatility of wind power system, which needs little data and simple calculating steps but has a large calculation error. The solution two uses the shape factor of 24 hours to calculate the energy loss and it needs the maximum data and the most complex calculating steps and gains a most accurate result among the four solutions. The solution three uses the shape factor expectation of whole year and its calculation error is little bigger than solution two. The solution four uses the shape factor expectation of the whole year to calculate the annual energy loss. Its calculation error is little bigger than solution two and three, but it only needs two values and once power flow calculation and the $7.73 \%$ is within the acceptable relative error range. If the environment around the wind power plant changes not particularly large, the shape factor expectation $K_{a v}=1.43$ can be used in the calculation of later years and it only needs the annual electric quantity one value to calculate the annual energy loss.

\section{Conclusion}

This paper proposes a new energy loss calculating method based on the shape factor to solve the problem of the traditional method that can't be used in large volatility system. The new method replaces the traditional representative day curve with the expectation curve of the calculation period and makes use of the shape factor of per hour to correct the expectation curve for a more accurate result. This method changes the calculating thinking of the energy loss that use the shape factor to replace the traditional empirical value. The normal system without wind power plant can also take account of this method to improve the calculating accuracy. Additionally, for the shape factor per hour of the wind power plant is within a small range, this paper proposes to use the annual shape factor expectation to calculate the annual energy loss directly. It at least only needs two values and once power flow calculation and its calculation error is within an acceptable range. As a result, this method has a strong engineering practicability.

\section{References}

1. Pierre Pinson, Henrik Madsen, Henrik Aa. Nielsen, George Papaefthymiou, Bernd Kl“ockl. From Probabilistic Forecasts to Statistical Scenarios of Short-term Wind Power Production[J]. Wind Energy (2008):1-13.

2. Pierre Pinson*, Henrik Aa. Nielsen, Jan K. Møller and Henrik Madsen, George N. Kariniotakis, Nonparametric probabilistic forecasts of wind powerrequired properties and evaluation $[\mathrm{J}]$. Wind Energy. 2007; 10:497-516.

3. Feng Ni. The Study on Calculation Method of Theoretical Energy Loss in Low-voltage Distribution Network[M].Xi'an: Xi'an University of Technology, 2008 (IN CHINESE).

4. Weiji, Zhou.Li, Loss Analysis of Areas with Wind Power System[M].Xinjiang University.2012.

5. Gao Hui. Loss Calculation and Measures Analysis of Distribution Grid[J]. Hunan Electric Power (2004).

6. Liao Xueqi. Line Loss Calculation Analysis and Loss Reduction Measures of Rural Power Network[M] Water Power Press, 2006. 